\title{
Photoinduced reversible switching of charge carrier mobility in conjugated polymers
}

\author{
M. Weiter ${ }^{1}$, J. Navrátil ${ }^{1, a}$, M. Vala ${ }^{1}$, and P. Toman ${ }^{1,2}$ \\ 1 Brno University of Technology, Faculty of Chemistry, Purkynova 118, 61200 Brno, Czech Republic \\ 2 Institute of Macromolecular Chemistry, AS CR, v.v.i., Prague, Czech Republic
}

Received: 29 August 2008 / Received in final form: 3 April 2009 / Accepted: 21 April 2009 Published online: 26 June 2009 - (c) EDP Sciences

\begin{abstract}
Photoinduced reversible switching of charge carrier mobility in conjugated polymers was studied by theoretical and experimental methods. The quantum chemical calculations showed that the presence of dipolar species in the vicinity of a polymer chain modifies the on-chain site energies and consequently increases the width of the distribution of hopping transport states. The influence of photoswitchable charge carrier traps on charge transport was evaluated by current-voltage measurement and by impedance spectroscopy method. It was found that deep traps switchable by photochromic reaction may significantly control the transport of charge carriers, which is exemplified as a significant decrease of the current and increase of parallel resistance measured by impedance spectroscopy.
\end{abstract}

PACS. 73.61.-r Electrical properties of specific thin films - 82.35.-x Polymers: properties; reactions; polymerization

\section{Introduction}

As an emerging area in organic electronics, polymer memories and switches have become an active research topic in recent years [1]. This paper deals with the concept of the photochromic switching of charge carrier transport in polymers. As the conductivity mechanism in these materials, a variable-range hopping in a positionaly random and energetically disordered system of localized states is widely accepted $[2,3]$. Over the last decades, hopping in random systems was extensively studied. Among these studies, the approach based on so-called effective transport energy level was shown to be especially efficient $[4,5]$. When the effective transport energy is established, the variable range hopping problem is virtually reduced to trap controlled transport model. According to this model, the transport of charge carriers in molecular solids is strongly influenced by the presence of centres capable of localizing charge carriers (traps). It was shown that deep traps may significantly affect the energy of the transport level and mobility of charge carriers [6] and thus control their transport.

Investigated polymer switch is based on switching of charge carrier mobility by photochromic species distributed in polymer matrix. Photochromic reactions, in addition to the changes in electronic spectra, are also accompanied by variations in refractive index, dielectric con-

\footnotetext{
a e-mail: xcnavratil@fch.vutbr.cz
}

stant, enthalpy, etc. [7]. Moreover, the reversible changes in physical or chemical properties of the photochromic species can be transferred to the microenvironment and supramolecular structure, and thus, can induce rich modifications in the surroundings. In a molecular solid build of non-polar polarizable units, e.g. polymer segments, containing a small amount of polar guest species, its dipole moment contributes to the field acting on surrounding molecules and modifies the local values of the polarization energy. This modification can cause a local decrease of the ionisation energy in an otherwise perfect crystal lattice, which represents a trap for hole. Thus, the presence of polar species may result in production of local states (charge traps) - in their vicinity; even thus they are not necessarily trapping sites themselves $[8,9]$. Reversible creation of such polar species can be obtained by e.g. suitable photochromic molecules. The induced change of electrostatic potential due to the charge-dipole interactions also shifts the site energies of individual polymer repeating units, and consequently the polymer transport levels are modified. Since the position and orientation of the additive with respect of the polymer chain are essentially random the effect results in broadening of the distribution of the transport states and consequently to the lowering of the charge carriers mobility. In the case of reversible formation and annihilation of such traps the electric charge transport can be even changed from space-charge limited to trap limited [10]. 

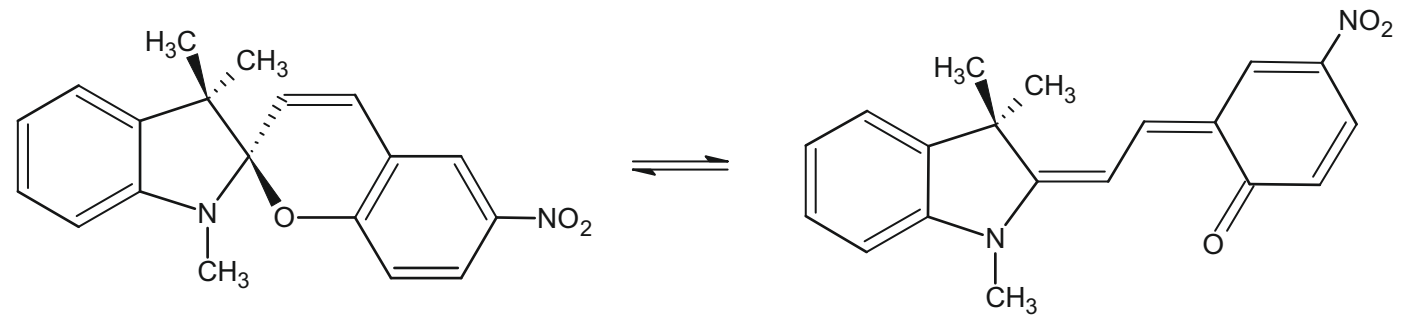

Fig. 1. Photochromic reaction of the spiropyran (left) to its metastable merocyanine form (right).

The purpose of the present work is to examine by quantum chemistry modeling and experimental characterization the optical and electrical switching properties of the suggested switch. For the study the $\pi$-conjugated photoconductive polymers poly[2-methoxy5-(2'-ethylhexyloxy)-p-phenylenevinylene] (MEH-PPV) doped by photochromic spiropyran 6-nitro-1',3',3', -trimethylspiro[2H-1-benzopyran-2,2'-indoline] (SP), which can be converted to a higher dipole moment possessing form referred to as (photo)merocyanine (MR), were used. The photochromic reaction of the spiropyran (SP) into (photo)merocyanine is depicted in Figure 1.

\section{Theoretical modeling}

In principle, the polar additive may influence the hole transport in two ways. First, if the highest occupied molecular orbital (HOMO) of the additive molecule is above the HOMO of the polymer, the additive molecules serve as energy level (chemical) traps. Second, the additive molecule possesses a large dipole moment that modifies the surrounding electrostatic potential landscape due to chargedipole interactions (dipolar traps) [11,12].

It was shown in our previous papers that SP/MR attached to the $\sigma$-conjugated poly[methyl(phenyl)silylene] creates chemical traps for holes $[11,13]$. However, the HOMO's of the $\pi$-conjugated polymers lie generally higher than the ones of the $\sigma$-conjugated polymers. The quantum chemical calculations show, that the HOMO of MEH-PPV is at least about $1 \mathrm{eV}$ above the HOMO's of SP and MR. For this reason we expect, that the additive does not create energy level traps in the investigated system, but influences the hole transport through the charge-dipole interactions.

The polymer chain is modeled by a sequence of $N=$ 4000 sites corresponding to the repeat units (alternating phenylenes and vinylenes). Each site $n$ is described by the energy $\varepsilon_{n}$ of a hole located at this site. The hole transport between the sites $n$ and $m$ is described by the transfer integral $b_{n, m}$. Because of linear character of the MEH-PPV chain and the size of the repeat units only the transfer integrals between the neighboring repeat units are important and the other can be neglected (tight-binding approximation). A typical value of the transfer integrals $b_{n, n+1}$ is about $1 \mathrm{eV}$, being significantly higher than the random disorder in site energies $\varepsilon_{n}$. This fact justifies the description of the on-chain hole motion using delocalized states approach rather than as hopping between localized states. Thus, hole motion on such a chain can be described by the Hamiltonian

$$
H=\sum_{n=1}^{N}\left[\varepsilon_{n} a_{n}^{+} a_{n}-b_{n, n+1}\left(a_{n+1}^{+} a_{n}+a_{n}^{+} a_{n+1}\right)\right],
$$

where $a_{n}$ and $a_{n}^{+}$are annihilation and creation operators of a hole at an $n$th site. Both quantities $\varepsilon_{n}$ and $b_{n, n+1}$ are influenced by the random structure of the polymer chain and its surrounding. The energy $\varepsilon_{n}$ is essentially equal to the negative of the first ionization potential of the corresponding repeat unit. Grozema et al. [14] developed a continuous disorder type model of the transfer integral $b_{n, n+1}$ distribution, showing that the hole mobility in a pure MEH-PPV is limited by the torsional disorder. We assume that the influence of the additives on the electronic coupling between the polymer repeat units is small in comparison with the electrostatic charge-dipole interaction modulating the site energies $\varepsilon_{n}$.

Polar species in the polymer chain vicinity modify the on-chain electrostatic potential due to the chargedipole interactions between a hole moving on the chain and dipole moments of individual additive molecules dispersed in the polymer. It is easy to show that the sum of these electrostatic potential changes shifts the hole site energies $\varepsilon_{n}$ by the value $\left\langle H O M O\left|\sum_{i} \Delta \phi_{i}\right| H O M O\right\rangle$, where $\Delta \phi_{i}$ are the changes of the electrostatic potential describing the charge-dipole interactions of a charge carrier localized at $|\mathrm{HOMO}\rangle$ with all surrounding polar additive molecules. The change of the shape of the highest molecular orbital $|\mathrm{HOMO}\rangle$ of the corresponding repeat unit induced by the additive is neglected (frozen orbital approximation) [11]. Since the positions and orientations of the additive molecules with respect to the polymer chain are essentially random, the effect results in broadening of the distribution of transport states. The most important parameter of this distribution (energetic disorder) is its halfwidth $\sigma\left(\varepsilon_{n}\right)$.

Recently, energetic disorder was numerically modeled by a dipolar lattice model [15]. This model considers a regular three-dimensional cubic lattice of sites being subject to periodic boundary conditions. A fraction of the sites is occupied by randomly oriented, immobile, and 
non-interacting dipoles. The electrostatic potential is then calculated for each site as a sum of the Coulombic potentials of all dipoles. This model was later analytically evaluated by Young [16]. He investigated the shape of the distribution and derived an equation for its half-width $\sigma$, being proportional to the dipole moment and the square root of the dipole concentration. Our model of the energetic disorder is a modification of this approach in order to take into account the linear shape of the polymer main chain, the size of its substituents, the absence of any well-ordered structure, and the fact, that the charge transport proceeds predominantly on the conjugated main chain. For this reason, the polymer chain was modeled as a line. Randomly oriented additive molecules, represented by point dipoles, were randomly placed in the vicinity of this line, but it was assumed that no additive molecule was placed at a distance shorter than $10 \AA$, which corresponds to the mean size of the polymer substituents and the size of the polar additive molecules. On the other hand, the influence of the additive molecules distant more than $50 \AA$ from the polymer chain was neglected. The additive molecules were placed also beyond the chain ends in order to ensure the homogeneity of the $\varepsilon_{n}$ distribution along the whole chain. The concentration of the additive was taken to be $c=4 \times 10^{-4} \AA^{-3}$. For each center representing a repeat unit, the energetic disorder was calculated as a sum of Coulombic electrostatic potentials from all additive molecules. With regard to the value of the minimal distance of an additive molecule from the chain the size of the polymer repeat units was neglected. The energetic disorder of $\varepsilon_{n}$ was calculated according to the abovedescribed model for several values of the dipole moment of the additive $m$. According to the central limit theorem [17], the resulting $\varepsilon_{n}$ distribution is a Gaussian-type distribution. For a typical value of $m=12 \mathrm{D}$ the halfwidth $\sigma\left(\varepsilon_{n}\right)=0.37 \mathrm{eV}$. Because the mutual interaction of the additive molecules is neglected, the half-width $\sigma\left(\varepsilon_{n}\right)$ is proportional to the dipole moment $m$ of the additive and to the square root of the additive concentration $\sqrt{c}$ just as in the dipolar lattice model [15]. However, the value of $\sigma\left(\varepsilon_{n}\right)$ calculated according to our model does not follow the relations derived by Young [16]. Furthermore it should be noted, that the $\varepsilon_{n}$ values show a strong site-to-site correlation between up to about 10th nearest neighboring sites. The correlation coefficient between the nearest neighbor $\varepsilon_{n}$ values is 0.97 . This fact can be explained by the long-range character of the charge-dipole interactions and the size of the MEH-PPV substituents hindering from close contact between additive molecules and the main chain. It should be pointed out, that the site-to-site correlation significantly affects the transport, thus the direct numerical calculation of the $\varepsilon_{n}$ values cannot be replaced by a simple generation of random numbers from a normal distribution.

The dipole moments of SP and MR forms calculated by the Hartree-Fock method are 5.5 and $11.9 \mathrm{D}$, respectively. These values suggest that the $\mathrm{SP} \rightarrow \mathrm{MR}$ reaction results in approximately doubling the energetic disorder. Moreover, one should take into account that, while the value obtained for SP is close to reality, the dipole moment of

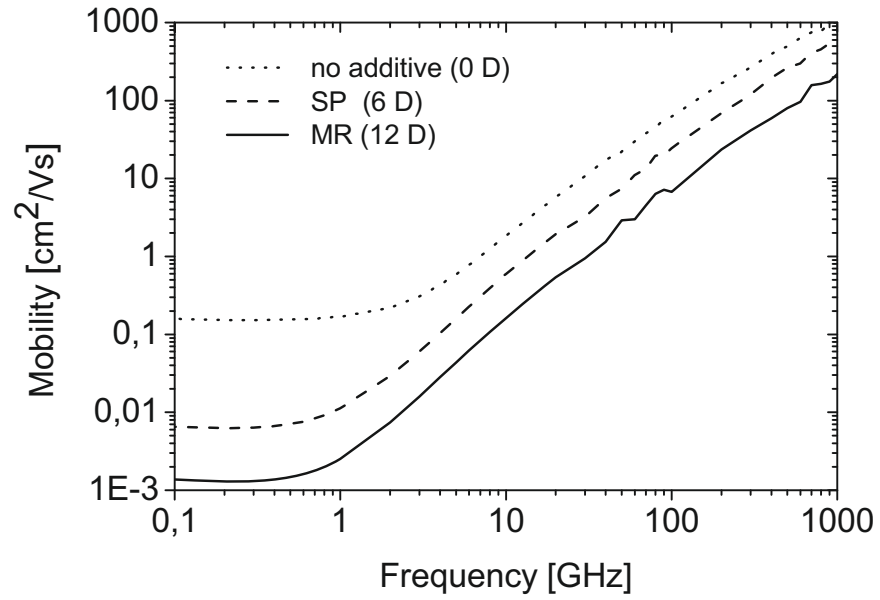

Fig. 2. The calculated frequency-dependent mobility for different additive dipole moments. The additive concentration was $c=4 \times 10^{-4} \AA^{-3}$.

MR is probably underestimated since the polar environment increases the zwitterionic character of MR. Bletz et al. [18] reported the dipole moment of MR measured in a polar environment to amount to $15 \div 20 \mathrm{D}$. Thus the real switching effect may be more effective than that estimated using the calculated values. The hole on-chain mobilities $\mu(\omega)$, calculated for different values of the additive dipole moments, are shown in Figure 2. All curves exhibit a saturation of $\mu(\omega)$ at low frequencies corresponding to the diffusive charge carrier motion in the long-time limit $(t>25 \mathrm{ps})$, and a rapid increase for higher frequencies related to the fast initial hole delocalization $(t<10 \mathrm{ps})$. This increase is mainly induced by the effective reduction of the number of disordered sites visited by a charge carrier during its oscillations induced by the electric filed. In both regions, the mobility decreases with the increasing additive dipole moment. It follows from Figure 2 that the change of the additive dipole moment from ca. $6 \mathrm{D}$ to $12 \mathrm{D}$ should result in an about five-fold decrease of the on-chain mobility. The effect would be even more pronounced if the experimental value of the MR dipole moment $(\approx 18 \mathrm{D}[13])$ is considered.

It should be noted that the results may be influenced by several other effects not taken into account by our model, namely dynamical fluctuations of both types of disorder and polaron formation. While the former effect results in a thermally activated diffusive charge carrier motion, the latter one increases charge localization. We assumed an ideal chemical structure, but the presence of polymerization defects decreases the on-chain mobility. The effect of the substituents was considered only in the modeling of the energetic disorder arising from the charge - additive dipole interaction. However, the presence of substituents has some influence also on the torsional and energetic disorder of undoped polymer chains. These phenomena will alter the mobility values but they should not significantly alter the mobility ratio since the photochromic mobility switching arises from the change of the levels of the static disorder. 


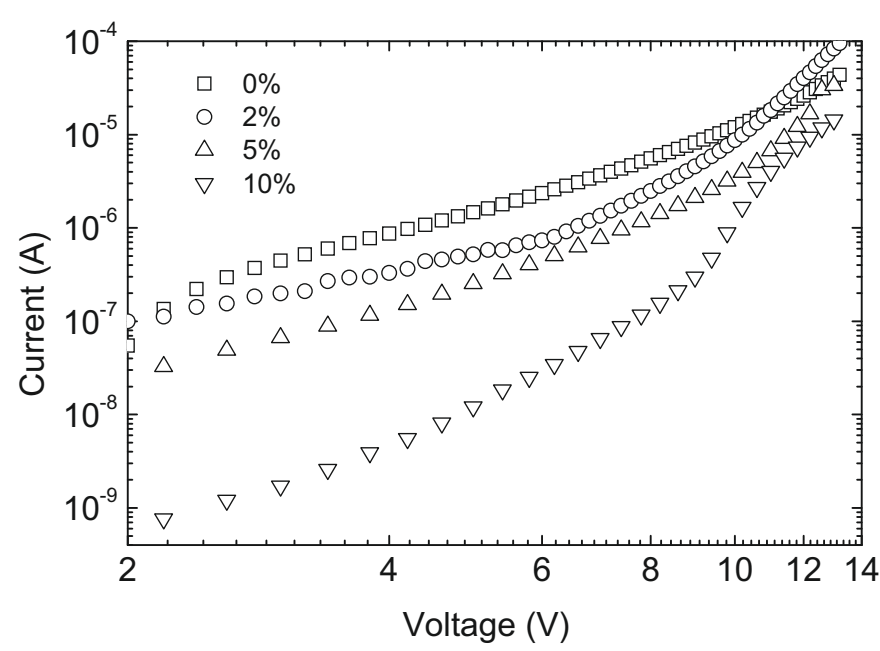

Fig. 3. Current-voltage characteristics for various concentration of spiropyran after the photochromic conversion induced by high photon dose corresponding to the merocyanine absorbance saturation.

\section{Experimental}

Polymer device was manufactured as a sandwich cell with a dielectric layer of MEH-PPV containing 0-30\% wt. of admixed SP. The active polymer layer was spin casted from chloroform solution on transparent indium tin oxide (ITO) electrode covering part of the glass substrate. The thickness of the active layer was about $150 \mathrm{~nm}$. The structure was completed by evaporation of aluminium top electrode typically $100 \mathrm{~nm}$ thick. Average electrode area that delimitates the active area of the device was $3 \mathrm{~mm}^{2}$. The photochromic reaction of spiropyrane was activated using a Nd:YAG pulse laser (1 $\mu \mathrm{J} /$ pulse) with third harmonic generation at $355 \mathrm{~nm}$ in order to measure the irradiation of the sample precisely. The electric response was studied by measuring the current-voltage $j(V)$ characteristics of the samples in the dark with Keithley 6517A electrometer. Dielectric properties were studied using a Hewlett Packard 4192A impedance analyzer. The measurements were performed in vacuum cryostat at room temperature.

\section{Results and discussion}

The photoswitching of charge carrier mobility was studied by standard current-voltage $j(V)$ measurement. The results for typical devices are shown as log-log plot in Figure 3 where the variation of the current at high irradiation dose corresponding to the merocyanine concentration saturation for different spiropyrane concentration is shown. In organic thin film devices the current is typically contact limited in low field region, whereas at higher field region space-charge or charge-trap limited conductivity are commonly accepted [19]. The results show this behavior. At low forward-bias voltages below $7-10 \mathrm{~V}$ the increase of $j$ with $V$ is relatively small, whereas in the higher field region the slope of the dependence is more pronounced, which is in accordance with Space-charge limited current (SCLC) theory. This theory proposes that the space charge which limits conduction is stored in the traps. In the case of energetically discrete trapping level, the SCL current can be expressed as

$$
j_{S C L}=\frac{9}{8} \varepsilon \varepsilon_{0} \mu \theta \frac{V^{2}}{L^{3}},
$$

where, $\varepsilon$ and $\varepsilon_{0}$ is the relative permittivity and permittivity of vacuum, $\mu$ is the charge carrier mobility, $V$ is the applied voltage, $L$ is the electrode distance and $\theta$ is the ratio of free to total charge carriers.

However, in cases of practical interest traps are usually distributed in energy. In that case traps will be filled from the bottom to the top of the distribution as applied electric field increases. This is equivalent to an upward-shift in the quasi-Fermi level with electric field. As a consequence, $\theta$ increases with electric field and the $j(V)$ characteristics becomes steeper. In terms of present work, the distribution of charge traps describes those induced by spiropyrane to merocyanine photochromic conversion. The presence of distribution of traps opens additional pathways to the relaxation of charge carriers towards steeper states. A zero order analytic description of the effect can be based on the Hoesterey and Letson formalism [20]. The latter is premised on the argument that the carrier mobility in a system with relative trap concentration $c$ is the product of the mobility in the trap-free system $\mu_{0}$ multiplied by trapping factor:

$$
\mu(c)=\mu_{0}\left[1+c \exp \left(\frac{E_{t}}{k T}\right)\right]^{-1},
$$

where $E_{t}$ is the energy of trapping level, $k$ is the Boltzmann constant and $T$ is the temperature. Consequently, the current flowing through the sample with enhanced number of trapping states will be less than in sample without traps. Figure 3, which shows the decrease of the photocurrent with increasing concentration of spiropyrane at high irradiation dose, proves this notion. Complementary, the decrease of the current with increasing photon dose demonstrated by number of laser pulses was observed. As the merocyanine concentration increase exponentially with photon dose and tends to saturate at high irradiation, the decrease of the current limits by about two orders of magnitude. In both cases the decrease is fully reversible after thermal fading in the dark or irradiation with a red light. The essential message is that the current thorough the irradiated device is significantly lowered by the presence of polar charge traps caused by photochromic conversion of spiropyrane to merocyanine. In another words the presence of traps lowers the mobility of charge carriers as expected from theoretical calculations.

To provide better insight into studied phenomena the real and complex part of the impedance $Z_{\text {Re }}$ and $Z_{\text {Im }}$ were recorded at test frequencies between 10 and $5 \times 10^{6} \mathrm{~Hz}$. The measured data were analyzed in the form of Cole-Cole diagram (real part of $Z$ vs. imaginary part of $Z$ ) wherein the frequency increases from right to left. The variations 
M. Weiter et al.: Photoinduced switching of charge carrier mobility in conjugated polymers

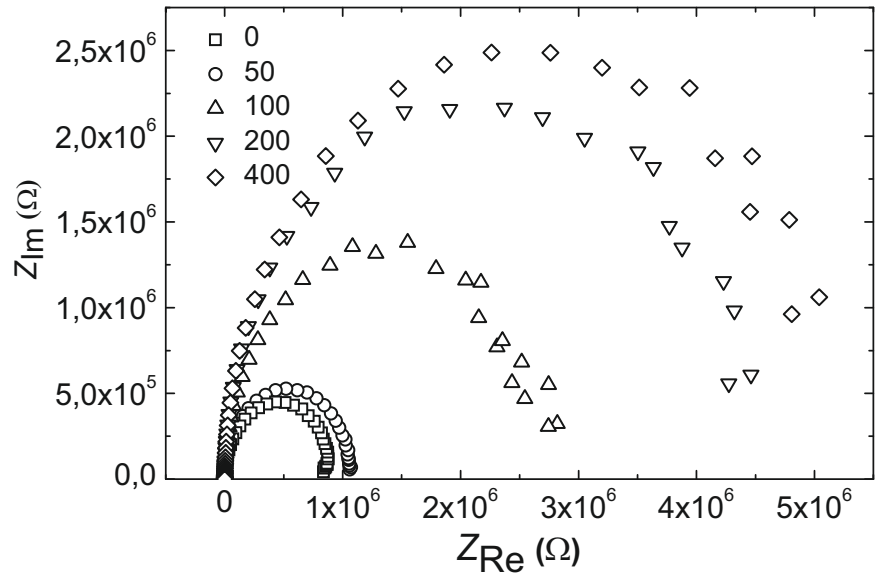

Fig. 4. Cole-Cole plot of the $20 \%$ mixture of the MEH-PPV:SP before ( 0 pulses) and after the photochromic conversion for four light doses expressed as a number of pulses $(1 \mu \mathrm{J} /$ pulse $)$.

of $Z_{\operatorname{Im}}$ with $Z_{\operatorname{Re}}$ as a function of photon dose at constant bias of $5 \mathrm{~V}$ for the device with $20 \%$ of spiropyrane are shown in Figure 4. All the $Z_{\mathrm{Im}}$ versus $Z_{\text {Re }}$ dependency shows a single semicircle which increases in size with increasing photon dose. This single semicircle could be fitted very well to a parallel combination of bulk resistance $R_{p}$ and capacitance $C_{p}$ in series with a resistance $R_{s}$, which is probably caused by the Ohmic contact at hole injecting ITO/MEH-PPV interface. From Figure 4 the bulk parallel resistance can be evaluated as the virtual intercept point of each Cole-Cole plot with $Z_{\mathrm{Re}}$ axis. Following this evaluation, Figure 4 clearly demonstrates the increasing parallel resistance of the sample with increasing photon dose, which is in accordance with previous results. The comparison of data obtained by impedance analysis and steady-state $j(V)$ characterization is depicted in Figure 5 for samples doped by $20 \%$ of spiropyrane after irradiation. Herein the results are related to the absorption of the samples at $590 \mathrm{~nm}$, which is proportional to merocyanine concentration as was described above. In this figure the left $y$-axis represents the relative increase of the parallel resistance obtained by impedance analysis, whereas the reverse value of the relative decrease of the current evaluated form the $j(V)$ characteristics is marked on right $y$-axis. It is shown that the dependencies which manifest the influence of merocyanine charge traps on the charge transport in MEH-PPV matrix are both almost linear.

\section{Conclusions}

The possibility of switching of charge carrier mobility was demonstrated. The quantum chemistry calculations showed that the presence of polar additive in the vicinity of polymeric chain modifies its transport levels. This increase in the energetic disorder eventuates in the decrease of the hole mobility. The change of the additive dipole moment from ca. $6 \mathrm{D}$ to $12 \mathrm{D}$, which corresponds to the studied photochromic reaction, results in an almost five-

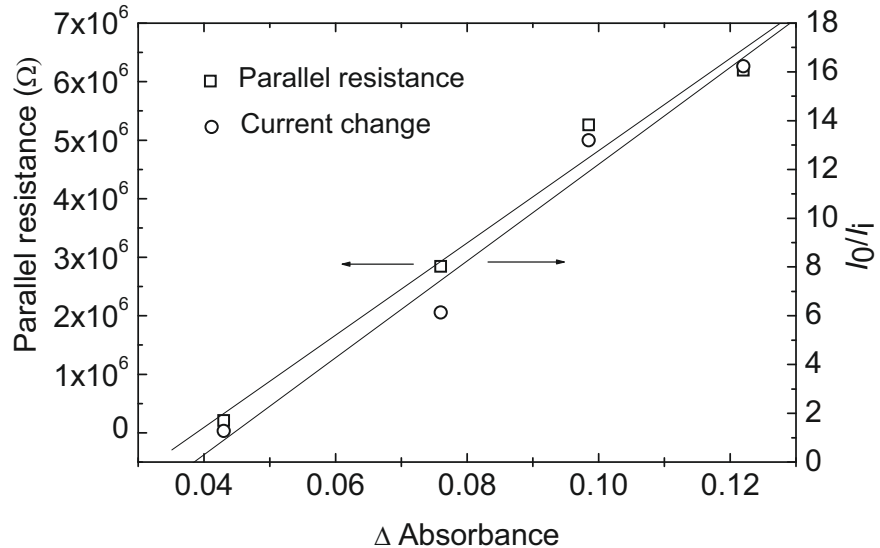

Fig. 5. The dependence of ratio of parallel resistance after the photochromic conversion to the value before conversion on the change of absorbance (left $y$-axis) and the ratio of the current before the photochromic conversion and after for different light dose at $4.2 \mathrm{~V}$ of the $20 \%$ mixture of the MEH-PPV:SP.

fold decrease of the on-chain mobility. The experimental behaviour of the system explored by means of currentvoltage characterization showed a significant decrease of the current thorough the sample after irradiation. The current decrease is proportional either to the concentration of spiropyrane in the sample or to the irradiation dose. The increase of parallel resistance of the sample with irradiation dose obtained by impedance analysis confirms this outcome. According to the trap controlled hopping model for the description of charge transport it was stated that the presence of new dipolar traps results in the decrease of the charge carrier mobility and thereby lowering the current as predicted by the theoretical calculations.

This work was supported by project KAN401770651 from the Academy of Sciences of the Czech Republic, project GA 203/06/0285 via the Czech Science Foundation and by project No. 0021630501 from Ministry of Education, Youth and Sport. A possibility of using computer time in MetaCenter is gratefully acknowledged.

\section{References}

1. G. Hadziioannou, G.G. Malliaras, Semiconducting polymers, Vols. 1 and 2 (Wiley-VCH, Weinheim, 2007)

2. M. Pope, C.E. Swenberg, Electronic Processes in Organic Crystals and polymers, 2nd edn. (Oxford University Press, Oxford, 1999)

3. H. Bässler, Phys. Stat. Sol. B 15, 175 (1993)

4. V.I. Arkhipov, E.V. Emelianova, G.J. Adriaenssens, Phys. Rev. B 64, 125125 (2001)

5. V.I. Arkhipov, P. Heremans, E.V. Emelianova, G.J. Adriaenssens, H. Bässler, J. Phys.: Condens. Matter 42, 9899 (2002)

6. V.I. Arkhipov, J. Ryenaert, Y.D. Jin, P. Heremans, E.V. Emelianova, G.J. Adriaenssens, H. Bässler, Synt. Met. 138, 209 (2003) 
7. H. Durr, H. Bouas-Laurent (Eds.), Photochromism: molecules and systems (Elsevier, Amsterdam, 2003)

8. S. Nešpùrek, J. Sworakowski, Thin Solid Films 393, 168 (2001)

9. M. Weiter, M. Vala, S. Nešpùrek, J. Sworakowski, O Salyk, O. Zmeškal, Mol. Cryst. Liq. Cryst. 430, 227 (2005)

10. P. Anderson, N.D. Robinson, M. Berggren, Adv. Mater. 17, $1798(2005)$

11. P. Toman, W. Bartkowiak, S. Nešpùrek, J. Sworakowski, R. Zaleśny, Chem. Phys. 316, 267 (2005)

12. F.L.E. Jakobsson, X. Crispin, M. Berggren, Organ. Electron. 10, 95 (2009)

13. P. Toman, S. Nešpùrek, W. Bartkowiak, J. Sworakowski, J. Lumin. 112, 386 (2005)
14. F.C. Grozema, P.T. van Duijnen, Y.A. Berlin, M.A. Ratner, L.D.A. Siebbeles, J. Phys. Chem. B 106, 7791 (2002)

15. A. Dieckmann, H. Bässler, P.M. Borsenberger, J. Chem. Phys. 99, 8136 (1993)

16. R.H. Young, Phil. Mag. B 72, 435 (1995)

17. J.A. Rice, Mathematical Statistics and Data Analysis, 2nd edn. (Duxbury Press, Belmont, 1995), ISBN 0-534-20934-3

18. M. Bletz, U. Pfeifer-Fukumura, U. Kolb, W. Baumann, J. Phys. Chem. A 106, 2232 (2002)

19. P.W.M. Blom, M.J.M. deJong, J.J.M. Vleggaar, Appl. Phys. Lett. 68, 3308 (1996)

20. D.C. Hoesterey, G.M. Letson, J. Phys. Chem. Solids 24, 1609 (1963)

To access this journal online: www.edpsciences.org 